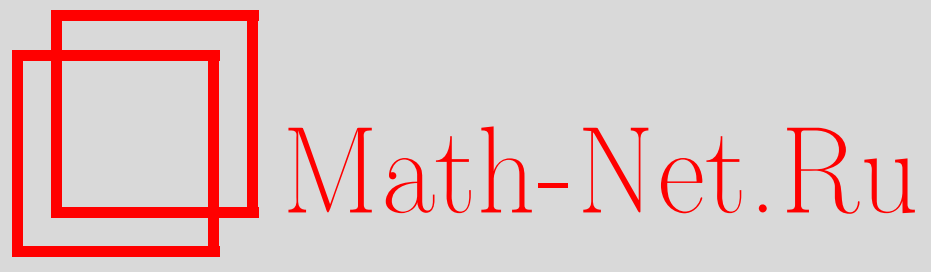

А. Н. Печень, Международный конгресс математиков 2018 года: от Рио-де-Жанейро к Санкт-Петербургу, УМН, 2018, том 73, выпуск 6, 211-217

DOI: https://doi.org/10.4213/rm9865

Использование Общероссийского математического портала Math-Net.Ru подразумевает, что вы прочитали и согласны с пользовательским соглашением http://www.mathnet.ru/rus/agreement

Параметры загрузки:

IP : 54.209 .52 .79

26 апреля 2023 г., 16:58:11

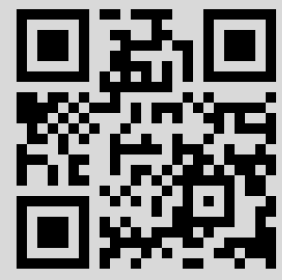




\section{Международный конгресс математиков 2018 года: от Рио-де-Жанейро к Санкт-Петербургу}

В период с 1 по 9 августа 2018 г. в Рио-де-Жанейро, Бразилия, прошел очередной, 28-й по счету, Международный конгресс математиков. Это крупнейшее математическое мероприятие, на котором вручается самая престижная в математике премия Филдса, а также премии Неванлинны, Гаусса, Черна, Лилавати. Конгрессы проводятся раз в четыре года начиная с 1897 г. (первый Конгресс проходил в Цюрихе, Швейцария). В них принимают участие несколько тысяч математиков со всего мира. В России Конгресс проводился только лишь в 1966 г., в Москве. Конгресс в Рио-де-Жанейро - первый в Южном полушарии.

\section{Генеральная ассамблея и определение Санкт-Петербурга как места проведения Конгресса 2022 года}

Конгрессу предшествовала Генеральная ассамблея Международного математического союза (ММС), которая прошла с 29 по 30 июля в Сан-Паулу, Бразилия. В Ассамблее принимают участие страны-участницы ММС. При этом каждая страна имеет квоту на определенное число делегатов и голосов. Ассоциированные члены МMC могут направлять для участия в Ассамблее только наблюдателей без права голоса. Остальные страны-участницы, в зависимости от группы, к которой они относятся, могут направить от одного до пяти делегатов с правом голоса. Россия относится к высшей, V группе. В этот раз в составе российской делегации были филдсовские лауреаты С. К. Смирнов и А. Ю. Окуньков, академики В. А. Васильев и С. В. Кисляков и ученый секретарь Национального комитета математиков РФ А.Н. Печень. В качестве наблюдателей в работе Ассамблеи принимали участие сопредседатель фонда "Сколково" А. В. Дворкович, профессор Нью-Йоркского университета Ф. А. Богомолов, доцент СПбГУ Ю. С. Белов.

На Ассамблее рассматривались вопросы, касающиеся развития математики в мире. Важной темой было определение места проведения следующих Конгресса и Ассамблеи, которые должны состояться в 2022 г. В этот раз впервые в истории на рассмотрение Генеральной ассамблеи были представлены две заявки - от Франции и от России.

В заявке от России в качестве места проведения Конгресса и Ассамблеи был предложен г. Санкт-Петербург. Подготовку и представление заявки в ММС осуществляла большая группа математиков под руководством С. К. Смирнова, Национальный комитет математиков РФ под руководством академиков Л.Д. Фаддеева (до 2017 г.) и В.В. Козлова (с 2017 г.). Заявка получила большую поддержку Правительства Российской Федерации и руководства Санкт-Петербурга.

Среди особенностей заявки - безвизовый въезд для всех участников Конгресса (аналог "паспорта болельщика" на чемпионате мира по футболу ФИФА-2018), оплата всех расходов, включая транспортные и расходы на проживание, для 1000 участников из развивающихся стран и для 1300 студентов, аспирантов и молодых ученых из стран СНГ [1]. Все это для того, чтобы облегчить математикам из разных стран

DOI: https://doi.org/10.4213/rm9865 
участие в Конгрессе, сделать его максимально доступным и открытым. Весь 2022 г. будет объявлен в России Годом математики, что было отмечено в заявке. В результате не только математическое сообщество России и мира, но и вся наша страна будет вовлечена в эти события.

Заявку от Франции готовил оргкомитет под руководством Франсуа Лозе (François Loeser). В качестве места проведения Конгресса был предложен Париж, в качестве места проведения Генеральной ассамблеи - Страсбург. Обе заявки были представлены в ММС до ноября 2016 г. В соответствии с правилами MMC предлагаемые места проведения Конгресса посещает специальный комитет (Site Visiting Committee), который затем формирует свои рекомендации и сообщает их Исполкому ММС. Визит этого комитета состоялся в марте 2017 г. В состав комитета вошли секретарь Математического союза Хелге Холден (Helge Holden) и члены Исполкома MMC - председатель организационного комитета Конгресса 2014 г. в Сеуле (Республика Корея) Хунджи Пак (Hyungju Park) и математик Кристиана Руссо (Christiane Rousseau). В период с 5 по 8 марта делегация находилась во Франции, с 8 по 11 марта - в России. По итогам визита на заседании Исполкома ММС в апреле 2017 г. было рекомендовано странам-участницам Ассамблеи поддержать заявку Российской Федерации.

Учитывая, что это решение Исполкома носит рекомендательный характер, Франция не сняла с рассмотрения свою заявку. В результате вопрос об определении места проведения Конгресса 2022 г. был вынесен на рассмотрение Генеральной ассамблеи в Сан-Паулу. Такая ситуация сложилась впервые за все время существования Международного математического союза. В истории Конгрессов ранее страны, заявки которых не были предварительно поддержаны Исполкомом ММС, снимали их с рассмотрения до Ассамблеи и, как правило, проводили Конгресс не через четыре, а через восемь лет.

Презентация обеих заявок состоялась 29 июля, в первый день Генеральной ассамблеи. На презентацию каждой заявки было отведено по 10 минут. Заявку от Франции представляли Мария Эстебан (María J. Esteban) и Франсуа Лозе, заявку от России Станислав Смирнов и Аркадий Дворкович. После представления заявок было отведено время для вопросов, а после перерыва состоялось голосование. За заявку Франции было подано 63 голоса, за заявку России - 83. В итоге Генеральная ассамблея приняла решение поддержать заявку России. Теперь очередной, 29-й по счету, Международный конгресс математиков пройдет в июле 2022 г. в Санкт-Петербурге, на площадке конгрессно-выставочного центра "Экспофорум" в историческом районе Пулковское. Генеральная ассамблея состоится либо в здании Двенадцати коллегий, либо в Таврическом дворце. По всей стране, а также в близлежащих странах пройдет несколько десятков сателлитных конференций, будет организован широкий круг мероприятий по популяризации математики. Кроме научных запланированы и эксклюзивные культурные мероприятия - в Эрмитаже, Мариинском и Михайловском театрах.

\section{Лауреаты премии Филдса 2018 года}

Через день после завершения Ассамблеи, в Рио-де-Жанейро начал свою работу Математический конгресс. Конгресс традиционно открылся объявлением лауреатов наиболее престижной премии для молодых математиков - премии Филдса. Премия названа в честь Джона Филдса, президента седьмого Конгресса (Торонто, 1924), который предложил на следующих Конгрессах награждать двух математиков золотой медалью в знак признания их заслуг. Теперь ее присуждают на каждом Конгрессе двум, трем или четырем математикам в возрасте не старше 40 лет (или достигшим 40-летия в год вручения премии). История присуждения медалей Филдса подробно изложена в статье С. П. Новикова [2].

На Конгрессе в Рио-де-Жанейро были вручены четыре премии Филдса. Лауреатами стали Кошер Биркар (Caucher Birkar), Акшай Венкатеш (Akshay Venkatesh), Алессио 
Фигалли (Alessio Figalli) и Петер Шольце (Peter Scholze) [3]. Состав Филдсовского комитета: Шигефуми Мори (Shigefumi Mori) - председатель, Элен Эсно (Hélène Esnault), Эдуард Файрайзл (Eduard Feireisl), Алис Гионнэ (Alice Guionnet), Найджел Хитчин (Nigel Hitchin), Джон Морган (John Morgan), Хи O (Hеe Oh), Андрей Окуньков, M. C. Рагунатан (M.S. Raghunathan), Кеннет А. Рибет (Kenneth A. Ribet), Терренс Tao (Terence Tao).

Кошер Биркар (род. 1978) - британский математик курдского происхождения, профессор Кембриджского университета. Ученик российского математика В. В. Шокурова. Специалист по бирациональной геометрии, внесший крупный вклад в теорию многообразий Фано и в программу минимальных моделей. В программе минимальных моделей доказал, вместе с К. Хэйконом (С. Насоn), Дж. Маккернаном (J. McKernan) и П. Касини (P. Cascini), конечную порожденность канонического кольца для многообразий общего типа. Одно из главных его достижений - доказательство, на основе результатов Хэйкона, Маккернана, Ч. Щу (С. Хи) и других, важной в алгебраической геометрии гипотезы учеников российского математика В. А. Исковских - А. А. Борисова, В. А. Алексеева, Л. А. Борисова о том, что комплексные многообразия Фано произвольной фиксированной размерности с $\epsilon$-логтерминальными особенностями для фиксированного положительного $\epsilon$ параметризуются (возможно, приводимым) алгебраическим многообразием. Награжден премией Филдса "за доказательство ограниченности многообразий Фано и вклад в исследование программы минимальных моделей" [4].

Акшай Венкатеш (род. 1981) - австралийский математик индийского происхождения, профессор Стэнфордского университета. Внес крупный вклад в ряд областей математики, включая теорию чисел, однородную динамику, арифметическую геометрию и теорию представлений, где решил множество давно стоявших проблем. Его работы в значительной мере связаны с работами ленинградского математика Юрия Владимировича Линника (1915-1972). Среди важных результатов Венкатеша, полученных им как индивидуально, так и вместе с соавторами, - результаты о равномерном распределении собственных функций Гекке-Маасса, оценка коэффициентов автоморфных форм, количественное решение задачи Фюрстенберга о некоторой динамической системе на отрезке, сильные и общие результаты, связанные с задачей Линника (1968) об описании представлений натуральных чисел в виде суммы трех квадратов. Венкатеш с соавторами широко использовал в качестве ключевого метода эргодический подход Линника в теории чисел, развивая и существенно дополняя его современной энтропийной техникой. Награжден премией Филдса за "синтез аналитической теории чисел, однородной динамики, топологии и теории представлений, позволивший разрешить давно стоявшие проблемы в таких областях, как равномерное распределение арифметических объектов" [5], [6].

Алессио Фигалли (род. 1984) - итальянский и швейцарский математик, профессор Высшей технической школы Цюриха. Специалист в области вариационного исчисления и дифференциальных уравнений в частных производных. Среди его достижений - ряд оптимальных или близких к оптимальности результатов о регулярности таких математических объектов, как отображения, реализующие наиболее эффективный перенос между заданными мерами; решения уравнений Монжа-Ампера (совместно с Гвидо де Филипписом (Guido De Philippis)); множества, близкие к оптимальности в классических геометрических неравенствах (совместно с рядом соавторов). А. Фигалли принадлежат пионерские работы о сингулярных множествах оптимальных отображений в теории переноса масс, а также новаторские приложения его общих результатов в теории семигеострофических уравнений, в задачах о мембранах, натянутых на препятствия, в задаче Стефана и в теории роста кристаллов. Награжден премией Филдса "за вклад в теорию оптимального переноса масс и ее приложения в области дифференциальных уравнений в частных производных, метрической геометрии и теории вероятностей" [7]. 
Петер Шольце (род. 1987) - немецкий математик, профессор Университета Бонна (Германия), самый молодой лауреат премии Филдса 2018 г. Область его исследований - арифметическая алгебраическая геометрия над $p$-адическими полями. Он разработал теорию перфектоидных пространств, которая позволяет связывать геометрию над $p$-адическими полями и геометрию в характеристике $p$, и применил эту теорию для доказательства, в случае полных пересечений, гипотезы Делиня о монодромии и весах. В качестве дальнейшего приложения он построил представления Галуа, связанные с элементами кручения в когомологиях локально симметричных пространств. Вместе с Б. Бхаттом (B. Bhatt) и M. Moppoy (M. Morrow) Шольце разработал целочисленную версию $p$-адической теории Ходжа, которая устанавливает связь между кручением в когомологиях Бетти и в кристаллических когомологиях. Также он существенно изменил некоторые разделы алгебраической топологии, относящиеся к топологическим гомологиям Хохшильда. Награжден премией Филдса "за преобразование арифметической алгебраической геометрии над $p$-адическими полями при помощи созданных им перфектоидных пространств, с приложениями в теории представлений Галуа, а также за развитие новых теорий когомологий” [8].

\section{Лауреаты премий Неванлинны, Гаусса, Черна, Лилавати}

Также на Конгрессе были объявлены лауреаты премии Неванлинны - за выдающиеся достижения молодым математикам в области информатики и вычислительной математики, премии Гаусса - за приложения математики в других областях, премии Черна - за выдающиеся достижения в математике и премии Лилавати - за выдающийся вклад в популяризацию математики.

Премия Неванлинны присуждается молодым (до 40 лет) математикам. В 2018 г. премия присуждена профессору Массачусетского технологического института Константиносу Даскалакису (Constantinos Daskalakis, род. 1981), специалисту в области теоретической информатики, алгоритмов, теории игр, алгоритмической теории игр, вычислительной биологии, прикладной теории вероятностей, - "за развитие понимания вычислительной сложности фундаментальных проблем, связанных с рынками, аукционами, равновесиями и другими экономическими структурами. Его работа предоставляет как эффективные алгоритмы, так и ограничения на то, что может быть выполнено эффективно в этих областях" [9].

Премия Гаусса вручается Международным математическим союзом совместно с Немецким математическим обществом за приложения математики в других областях, включая технологии, бизнес и повседневную жизнь, в том числе за практические приложения. В 2018 г. премия была присуждена профессору Стэнфордского университета Дэвиду Донохо (David Donoho, род. 1957) “за фундаментальный вклад в математический, статистический и вычислительный анализ важных проблем в теории передачи сигналов". Важнейшие результаты Дэвида Донохо находятся в области разработки курвлетов (специализированных вариантов вейвлетов) и в теории compressive sensing [10].

Премия Черна присуждается за выдающиеся достижения в математике без какихлибо возрастных ограничений. В 2018 г. премия была присуждена японскому математику, профессору Киотского университета Масаки Кашиваре (Masaki Kashiwara, род. 1947) "за его выдающийся и основополагающий вклад в алгебраический анализ и теорию представлений в течение почти 50 лет". Масаки Кашивара внес фундаментальный вклад в создание и развитие алгебраического анализа, в теорию $D$-модулей, теорию Ходжа, теорию пучков и теорию представлений [11].

Премия Лилавати присуждается за выдающийся вклад в популяризацию математики. В 2018 г. премия была присуждена Али Несину (Ali Nesin, род. 1957) за выдающийся вклад и крупные достижения по повышению внимания к математике в Турции, в особенности за неустанную работу по созданию и развитию "Математической деревни" - особого места, в котором каждый может заниматься изучением, исследованиями и применениями математики [12]. 


\section{Пленарные и секционные выступления на Конгрессе 2018 года}

В первый день Конгресса, после объявления лауреатов премий, с представлением их работ выступили (в хронологическом порядке): Ėva Tardos (США) - о работах Константиноса Даскалакиса; Michael Rapoport (Германия) - о работах Петера Шольце; Peter Sarnak (США) - о работах Акшая Венкатеша; Luis Cafarelli (США) о работах Алессио Фигалли; Christopher Hacon (США) - о работах Кошера Биркара; Emmanuel Candes (США) - о работах Дэвида Донохо; Pierre Schapira (Франция) о работах Масаки Кашивары; Gert-Martin Greuel (Германия) - об Али Несине и его "Математической деревне".

Сами лауреаты в дальнейшем также выступили с рассказами о своих результатах. Кроме того, были прочитаны две специальные лекции - Эмми Нётер и Абелевская. Лекцию Эмми Нётер по теме "Conformal geometry on 4-manifolds" 3 августа прочитала китайский и американский математик Sun-Yung Alice Chang. Майкл Атья (Michael Atiyah, Великобритания) 6 августа прочитал вызвавшую большой интерес Абелевскую лекцию "The future of Mathematical Physics: new ideas in old bottles" [13]. В частности, М. Атья анонсировал доказательство гипотезы Римана о нулях дзета-функции и вычисление постоянной тонкой структуры в квантовой электродинамике с использованием некоммутативного обобщения формулы Эйлера $e^{2 i \pi}=1$ и функций Тодда. Эти утверждения в дальнейшем вызвали разноречивые мнения среди математиков, в значительной мере скептические.

Каждый день Конгресса, кроме дня открытия 1 августа и свободного дня 5 августа, начинался с трех либо четырех пленарных лекций длительностью каждая по часу. Также некоторые пленарные лекции читались вечером. Параллельные секции в это время не проводились. Всего было прочитано 38 лекций, включая восемь представлений в день открытия, восемь лекций лауреатов и две специальные лекции.

Пленарным докладчиком, представляющим Россию и США, выступил филдсовский лауреат А. Ю. Окуньков (Институт проблем передачи информации им. А. А. Харкевича РАН и Высшая школа экономики/Колумбийский университет) - с лекцией "On the crossroads of enumerative geometry and geometric representation theory".

Перечень пленарных лекций, не включая уже перечисленные выше представления лауреатов и две специальные лекции, выглядит следующим образом (символом * отмечены лекции лауреатов премий).

\section{1 августа}

* Constantinos Daskalakis (USA) "Equilibria, fixed points, and computational complexity"

2 августа

Simon Donaldson (UK/USA) "Some recent developments in Kähler geometry and exceptional holonomy"

Sylvia Serfaty (France/USA) "Systems of points with Coulomb interactions"

Rahul Pandharipande (Switzerland) "Geometry of the moduli space of curves"

\section{3 августа}

Andrei Okounkov (Russia/USA) "On the crossroads of enumerative geometry and geometric representation theory"

Gregory Lawler (USA) "Conformally invariant measures on paths and loops"

Carlos Gustavo Moreira (Brazil) "Dynamical systems, fractal geometry and diophantine approximations"

* Akshay Venkatesh (USA) "Cohomology of arithmetic groups"

\section{4 августа}

Luigi Ambrosio (Italy) "Calculus, heat flow and curvature-dimension bounds in metric measure spaces"

Lai-Sang Young (USA) "Dynamical systems evolving"

* Peter Scholze (Germany) "Period maps in p-adic geometry"

* Alessio Figalli (Switzerland) "Regularity of interfaces in phase transitions via obstacle problems" 


\section{6 августа}

Ronald Coifman (USA) "Harmonic analytic geometry on subsets in high dimensions Empirical models"

Peter Kronheimer (USA), Tomasz Mrowka (USA) "Knots, three-manifolds and instantons"

Catherine Goldstein (France) "Long-term history and ephemeral configurations"

* Caucher Birkar (UK) "Classification of algebraic varieties"

\section{7 августа}

Alexander Lubotzky (Israel) "High dimensional expanders"

Nalini Anantharaman (France) "Delocalization of Schrödinger eigenfunctions" Sanjeev Arora (USA) "The mathematics of machine learning and deep learning"

* David Donoho (USA) "Compressed sensing - from blackboard to bedside"

\section{8 августа}

Assaf Naor (USA) "Metric dimension reduction: A snapshot of the Ribe program" Geordie Williamson (Australia/Germany) "Representation theory and geometry" Christian Lubich (Germany) "Dynamics, numerical analysis and some geometry"

* Masaki Kashiwara (Japan) "Crystal bases and categorifications"

* Ali Nesin (Turkey) "The adventure of the Mathematics Village"

\section{9 августа}

Gil Kalai (Israel) "Noise stability, noise sensitivity and the quantum computer puzzle" Michael Jordan (USA) "Dynamical, symplectic and stochastic perspectives on gradientbased optimization"

Vincent Lafforgue (France) "Global Langlands parameterization and shtukas for reductive groups"

Н.Н. Андреев (Математический институт им. В.А. Стеклова РАН) совместно с Симоном Пампеной (Simon Pampena, Австралия), Роджерио Мартинсом (Rogério Martins, Португалия), Марианой Перейрой (Mariana Pereira, Уругвай) и Тадаши Токиедой (Tadashi Tokieda, США) принял участие в панельной дискуссии "New avenues for raising public awareness of mathematics" в секции "Математическое образование и популяризация математики".

Перечень приглашенных секционных докладчиков, представляющих Россию (в порядке, соответствующем порядку секций в программе):

И.А. Панин (Санкт-Петербургское отделение Математического института им. В. А. Стеклова РАН и Исследовательская лаборатория им. П. Л. Чебышёва СПбГУ) с докладом "On Grothendieck-Serre conjecture concerning principal bundles" в секции "Алгебра";

А. А. Логунов (Исследовательская лаборатория им. П. Л. Чебышёва СПбГУ, ТельАвивский университет и Принстонский Институт перспективных исследований, Россия/Израиль/США), совм. с Е. В. Малинниковой (Норвегия) с докладом "Quantitative propagation of smallness for solutions of elliptic equations" в секциях "Геометрия" и "Уравнения в частных производных";

M. В. Финкелъберг (Институт проблем передачи информации им. А. А. Харкевича РАН, Высшая школа экономики и Сколковский институт науки и технологий) с докладом "Double affine Grassmannians and Coulomb branches of 3-dimensional $N=4$ quiver gauge theories" в секции "Теория Ли и обобщения";

Д. С. Челкак (École Normale Supérieure и Санкт-Петербургское отделение Математического института им. В.А. Стеклова РАН, Франция/Россия) с докладом "Planar Ising model at criticality: State-of-the-art and perspectives" в секциях "Анализ и операторные алгебры" и "Вероятность и статистика";

А.А. Белавин (Институт теоретической физики им. Л. Д. Ландау РАН, Институт проблем передачи информации им. А. А. Харкевича РАН и Московский физико-технический институт) с докладом "Special geometry on Calabi-Yau moduli spaces and $Q$-invariant Milnor rings" в секции "Математическая физика". 
Благодарим В. М. Бухштабера, И. В. Воловича и Д. О. Орлова за ценные комментарии, С.О. Горчинского, А.Н. Соболевского, И. Д. Шкредова, К. А. Шрамова за существенную помощь и замечания о работах филдсовских лауреатов П. Шольце, А. Фигалли, А. Венкатеша, К. Биркара соответственно.

По поручению Национального комитета математиков РФ Ученый секретарь Национального комитета математиков РФ А.Н. Печень

\section{Список литературы}

[1] Официальный сайт заявки Российской Федерации на проведение Международного конгресса математиков 2022 года в г. Санкт-Петербурге http://icm2022.ru/

[2] С. П. Новиков, "Медали Филдса", УМН, 65:6(396) (2010), 200-203; англ. пер.: S. P. Novikov, "Fields Medals", Russian Math. Surveys, 65:6 (2010), 1199-1203.

[3] Fields Medals 2018

https://www.mathunion.org/imu-awards/fields-medal/fields-medals-2018

[4] Fields Medal. Caucher Birkar Citation https://www.mathunion.org/fileadmin/IMU/Prizes/Fields/2018/Birkar-Citation.pdf

[5] Fields Medal. Akshay Venkatesh Citation https://www.mathunion.org/fileadmin/IMU/Prizes/Fields/2018/Venkatesh-Citation.pdf

[6] И. Шкредов, “Эргодический импульс и синтез идей”, Троицкий вариант, № 260, 14.08.2018, c. 3

[7] Fields Medal. Alessio Figalli Citation https://www.mathunion.org/fileadmin/IMU/Prizes/Fields/2018/Figalli-Citation.pdf

[8] Fields Medal. Peter Scholze Citation https://www.mathunion.org/fileadmin/IMU/Prizes/Fields/2018/Scholze-Citation.pdf

[9] Rolf Nevanlinna Prize. Constantinos Daskalakis Citation https://www.mathunion.org/imu-awards/rolf-nevanlinna-prize/rolf-nevanlinna-prize-2018; https://www.mathunion.org/fileadmin/IMU/Prizes/Nevanlinna/Daskalakis-Citation.pdf

[10] Carl Friedrich Gauss Prize. David L. Donoho Citation https://www.mathunion.org/imu-awards/carl-friedrich-gauss-prize/carl-friedrich-gaussprize-2018; https://www.mathunion.org/fileadmin/IMU/Prizes/Gauss/Donoho-Citation.pdf

[11] Chern Medal Award. Masaki Kashiwara Citation https://www.mathunion.org/imu-awards/chern-medal-award/chern-medal-award-2018 https://www.mathunion.org/fileadmin/IMU/Prizes/Chern/Kashiwara-citation.pdf

[12] Leelavati Prize 2018. Ali Nesin Citation https://www.mathunion.org/imu-awards/leelavati-prize/leelavati-prize-2018 https://www.mathunion.org/fileadmin/IMU/Prizes/Leelavati/Nesin-Citation.pdf

[13] Abel Lecture - The future of Mathematical Physics: new ideas in old bottles M. Atiyah - ICM2018 https://www.youtube.com/watch?v=fUEvTymjpds 